\title{
Femtosecond gain characteristics of the discharge-pumped ArF excimer amplifier
}

\author{
K. Mossavi, Th. Hofmann, G. Szabó, and F. K. Tittel \\ Department of Electrical and Computer Engineering, Rice Quantum Institute, Rice University, P.O. Box 1892, Houston, Texas 77251
}

Received October 8, 1992

\begin{abstract}
The gain characteristics of a discharge-pumped ArF excimer amplifier are measured with $\sim 700$-fs-long pulses. The small-signal gain and saturation energy are found to be $0.17 \mathrm{~cm}^{-1}$ and $3.65 \pm 0.15 \mathrm{~mJ} / \mathrm{cm}^{2}$, respectively. The maximum output energy density extracted from the deeply saturated amplifier is as much as $10 \mathrm{~mJ} / \mathrm{cm}^{2}$. The demonstrated femtosecond gain characteristics of ArF indicate that, by utilizing sufficiently high seed pulse energies, the ArF excimer laser is expected to show a performance similar to a femtosecond high-brightness $\mathrm{KrF}$ excimer laser system.
\end{abstract}

In recent years we have witnessed fundamental progress in high-brightness ultrashort-pulse excimer laser technology. High-power femtosecond gas laser systems have been developed based on $\mathrm{XeCl},{ }^{1} \mathrm{KrF},{ }^{2}$ and, very recently, $\mathrm{XeF}(\mathrm{C} \rightarrow \mathrm{A})$ (Ref. 3) excimers. The ultrashort-pulse ArF excimer laser systems, however, have so far shown significantly lower performance. ${ }^{4-7}$ This can be explained by two main reasons. On one hand, the seed pulse generation becomes increasingly difficult since the necessary wavelength $(193 \mathrm{~nm})$ lies outside the range that is covered by currently available frequency-doubling crystals; whereas, on the other hand, the losses of the optical materials increase with decreasing wavelength. The design of the high-power ArF laser systems was further impeded by the fact that, to the best of our knowledge, the saturation energy density, which is the most important design and scaling parameter of such systems, has not been published in the femtosecond regime.

In this Letter we present the gain characteristics of a discharge-pumped $\mathrm{ArF}$ excimer amplifier measured with $\sim 700$-fs probe pulses generated by a newly developed, spectrally compensated sum-frequency mixing scheme ${ }^{8}$ and demonstrate that the femtosecond ArF amplifier can provide the same high performance as a $\mathrm{KrF}$ amplifier.

The experimental apparatus is depicted in Fig. 1. The seed pulses are generated by using 50-ps 266-nm pulses from a Nd:YAG laser and 300-fs 707-nm pulses from a dye laser. ${ }^{9}$ The two beams enter the dispersively compensated frequency-mixing scheme, ${ }^{8}$ where their sum frequency at $193 \mathrm{~nm}$ is generated. By using 3-mJ pulse energy at $266 \mathrm{~nm}$ and $0.8 \mathrm{~mJ}$ at $707 \mathrm{~nm}$, the pulse energy at $193 \mathrm{~nm}$ was $12 \mu \mathrm{J}$, with a typical spectral width of $0.2 \mathrm{~nm}$, corresponding to an approximately 300 -fs pulse duration.

The energy of the seed pulses was too low to allow for direct pulse-duration measurements. Instead, the pulse duration was measured after doublepass amplification in an ArF excimer laser, with a Michelson-type autocorrelator based on two-photon ionization of $\mathrm{Xe}$ and was found to be $710 \mathrm{fs}$, assuming a Gaussian pulse shape. Since the corre- lation measurements were performed after the pulses traveled four times through the 5-mm-thick $\mathrm{CaF}_{2}$ window of the laser and approximately $8 \mathrm{~m}$ in air, one expects noticeable temporal pulse broadening with respect to the seed pulse duration. Considering that the seed pulse duration is expected to be the same as that of the 707-nm pulse (i.e., $300 \mathrm{fs}$; Ref. 8), the assumed initial and the measured final pulse durations are in good agreement with the values reported in Ref. 6 under similar conditions. Considering the supporting experimental evidence presented in Ref. 6 , we believe that the main pulsebroadening mechanism is coherent pulse propagation through $\mathrm{O}_{2}$. In this case, taking into account the effective optical path lengths that the pulses traveled through air between the seed pulse generator and the excimer amplifier and between the amplifier and the autocorrelator, the actual probe pulse duration in the amplifier is expected to be approximately $500 \mathrm{fs}$.

To obtain energies high enough to reach deep saturation in the gain measurements, the seed pulses were preamplified in a single pass through the excimer laser. A Lambda Physik EMG-101-type excimer laser rated at $200-\mathrm{mJ}$ maximum output energy was used in the measurements. After single-pass amplification the total energy was typically $500 \mu \mathrm{J}$.

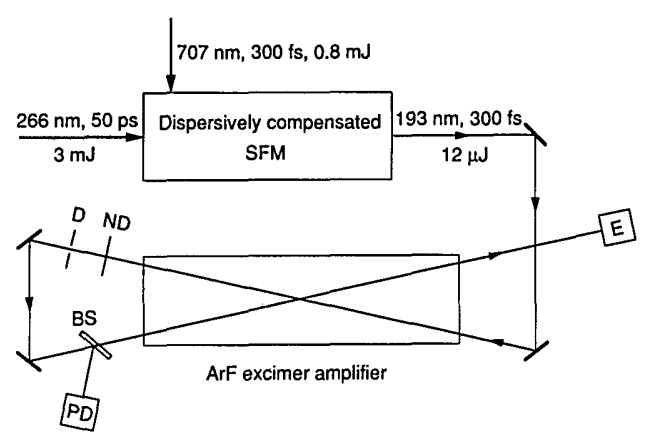

Fig. 1. Experimental arrangement for measurements of the femtosecond gain characteristics of ArF. PD, photodiode; $\mathrm{E}$, energy meter; $\mathrm{D}$, diaphragm; ND, neutral-density filter; BS, beam splitter; SFM, sum-frequency mixer. 
To avoid difficulties in the gain measurements caused by spatial inhomogeneities of the probe beam, ${ }^{3}$ the most homogeneous central part of the spot containing $50 \mu \mathrm{J}$ of energy was selected by a diaphragm with a $3-\mathrm{mm}$ diameter.

After the diaphragm and the turning mirrors, a small portion of the beam was coupled out with a $\mathrm{CaF}_{2}$ plate and sent onto a solar-blind vacuum photodiode (Hamamatsu R1193U-04) that provided the input energy reference for the gain measurements. Since possible nonlinear optical losses in the $\mathrm{CaF}_{2}$ beam-splitter plate and the absorption in air can lead to measured input energies that are higher than the actual energy entering the amplifier, we calibrated the input energy reference by measuring the energy directly in front of the input window of the excimer laser relative to the photodiode signal. To provide the dynamic range that is necessary for the input energy measurements we also used neutral-density filters in front of the photodiode. These neutraldensity filters had been calibrated individually by using an energy meter and the femtosecond pulses from the ArF laser. The input-output energy characteristics of the $\mathrm{ArF}$ amplifier were obtained by measuring the energy of the beam after the second pass through the amplifier with an energy meter (Laser Precision RJ 7620) equipped with a type RJP-735 detector head.

The use of the diaphragm was also important to minimize the amplified spontaneous emission (ASE) in the probe pulse. With the diaphragm in place, the time-integrated ASE energy barely reached the sensitivity threshold of the energy meter; consequently, only an upper limit of $0.2 \%$ could be obtained.

A clearly visible blue fluorescence indicated the presence of the intensity-dependent losses in the window materials. ${ }^{10-12}$ These losses, however, may distort the measured gain characteristics. To account for this effect we measured the transmission of a $\mathrm{CaF}_{2}$ window similar to the ones used in the excimer laser. For the transmission measurements, the $\mathrm{CaF}_{2}$ sample was inserted into the laser beam close to the output window where the pulses exit the amplifier after the second pass. In this way, the intensity on the sample was the same as on the output window. The measured transmission as a function of the energy density is shown in Fig. 2. From this figure we can see that the output intensity range used for the gain measurements extends only up to the onset of the nonlinear processes; i.e., the transmission of the $\mathrm{CaF}_{2}$ plate decreases only slightly from its initial value of $85 \%$ to $70 \%$ at the highest intensity. For comparison, the transmission as measured with nanosecond pulses when no nonlinear effects are expected is indicated by a square on the vertical axis. Although there is some controversy in the literature as to whether the main nonlinear loss mechanism in $\mathrm{CaF}_{2}$ at $248 \mathrm{~nm}$ is two-photon or three-photon absorption, ${ }^{11,12}$ considering the $10-\mathrm{eV}$ band gap of $\mathrm{CaF}_{2}$, at $193 \mathrm{~nm}$ the nonlinear losses are expected to be due to two-photon absorption. Assuming that the overall losses are small, i.e., $\alpha_{1} L, \alpha_{2 p} L I_{0}{ }^{2} \ll 1$ (where $\alpha_{1}$ and $\alpha_{2 p}$ are the linear and two-photon absorption coefficients of $\mathrm{CaF}_{2}$, respectively, $L$ is the thickness of the sample, and $I_{0}$ is the peak intensity on the sample), the transmission, defined as the ratio of the energy of the pulse entering and leaving the $\mathrm{CaF}_{2}$ plate, can be written as

$T=\frac{\int_{-\infty}^{\infty}\left[I_{0} A(t)-\alpha_{1} L I_{0} A(t)-\alpha_{2 p} L I_{0}^{2} A^{2}(t)\right] \mathrm{d} t}{\int_{-\infty}^{\infty} I_{0} A(t) \mathrm{d} t}$,

where $A(t)$ is the pulse envelope. From Eq. (1) we expect a linear decrease of the transmission with increasing peak intensity. The measured curve clearly indicates this tendency. The solid curve in Fig. 2 represents the best linear fit to the data points. This curve was used to correct the measured gain data for window transmission. Furthermore, by using Eq. (1) and assuming a Gaussian pulse shape, the slope of the line provides an approximate value of $3.1 \times 10^{-11} \mathrm{~cm} / \mathrm{W}$ for the two-photon absorption coefficient of $\mathrm{CaF}_{2}$ at $193 \mathrm{~nm}$. It should be noted, however, that the intensity-dependent transmission can be different with samples from different suppliers. The losses in this pulse duration range seem to be tolerable, but with decreasing pulse duration they may become prohibitive. Minimizing these losses by using different optical materials such as $\mathrm{BaF}_{2}$ (Ref. 6) is a critical issue in the design of high-power short-pulse ArF laser systems.

The measured output energy density of the ArF excimer amplifier as a function of the input energy density can be seen in Fig. 3. Since the small-signal gain depends on the timing of the seed pulse with respect to the excimer amplifier, the shot-to-shot timing jitter, if it were large enough to cause a noticeable (shot-to-shot) change in the small-signal gain, introduces some uncertainty in the gain characteristics. This uncertainty can be decreased by averaging a sufficiently large number of shots (in the figure each point represents an average of 100 shots), and the reliability of the gain curves can additionally be checked by performing measurements for different temporal delays. Accordingly, the measurements were made for two different time delays. The experimental points represented by circles were obtained for operating conditions such that the seed

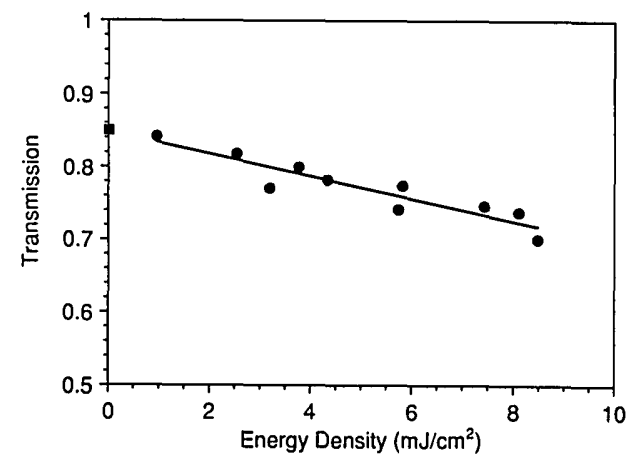

Fig. 2. Intensity-dependent transmission of a $\mathrm{CaF}_{2}$ laser window at $193 \mathrm{~nm}$. The filled square on the vertical axis indicates the transmission measured by a nanosecond pulse. 


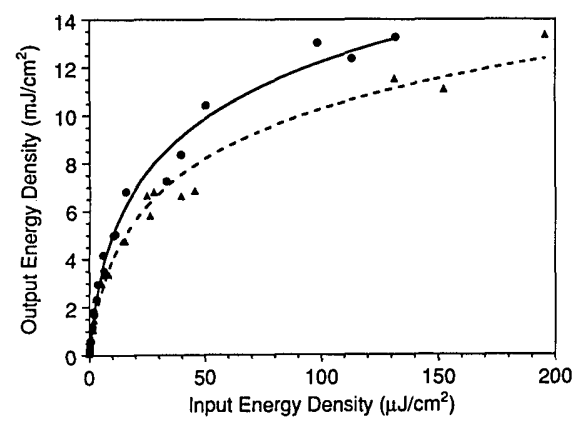

Fig. 3. Input-output energy characteristics of the ArF amplifier. The circles and triangles represent data for two different time delays of the probe pulse with respect to the amplifier. The solid and dashed curves indicate the best Frantz-Nodvik fits to the experimental data.

pulse entered the amplifier when its ASE, and consequently the gain, was at a maximum. The triangles represent data belonging to a temporal delay that is larger by $4 \mathrm{~ns}$ (which is two times the 2-ns timing jitter), corresponding to a situation when the intensity of the ASE decreased to approximately half of its maximum value. Both the input and output energy values are corrected for window transmission by using the transmission curve in Fig. 2. The solid and dashed curves in Fig. 3 represent the best Frantz-Nodvik fits to the experimental points by using the expression given as Eq. (1) in Ref. 13. The respective best-fit parameters for the small-signal gain coefficient and saturation energy are $0.17 \mathrm{~cm}^{-1}$ and $3.8 \mathrm{~mJ} / \mathrm{cm}^{2}$ (circles and solid curve) and 0.16 $\mathrm{cm}^{-1}$ and $3.5 \mathrm{~mJ} / \mathrm{cm}^{2}$ (triangles and dashed curve).

The small-signal gain coefficients of 0.17 and 0.16 $\mathrm{cm}^{-1}$ correspond to small-signal gains of 934 and 626, respectively, considering the $40-\mathrm{cm}$ gain length of the excimer laser. A comparison of the measured smallsignal gains and saturation energies shows that for the time delays used, the small-signal gain differs considerably, whereas the saturation energy, as expected, remains the same within the experimental accuracy. This also demonstrates the reliability of the measured saturation energy values.

The saturation energy density of $3.65 \pm 0.15$ $\mathrm{mJ} / \mathrm{cm}^{2}$ obtained for ArF is considerably higher than the value of $2.0 \mathrm{~mJ} / \mathrm{cm}^{2}$ reported in Ref. 14 for $\mathrm{KrF}$. This is somewhat surprising considering that the two molecules are spectroscopically rather similar. ${ }^{15}$ By using the stimulated emission cross sections given in Ref. 15, the calculated energy densities are also similar; 3.6 and $4.2 \mathrm{~mJ} / \mathrm{cm}^{2}$ for $\mathrm{ArF}$ and $\mathrm{KrF}$, respectively. The difference in the measured values is mainly due to the fact that the values presented here are corrected for window transmission. The saturation energy density is $2.3 \mathrm{~mJ} / \mathrm{cm}^{2}$ for $\mathrm{ArF}$, if uncorrected data are used, which is close to the value for $\mathrm{KrF}$.

A comparison of the femtosecond gain characteristics of $\mathrm{KrF}$ and $\mathrm{ArF}$ and the recently measured gain recovery time of ArF (Ref. 16) indicates that, by utilizing sufficiently high seed pulse energies, the $\mathrm{ArF}$ excimer laser is expected to show a performance similar to a femtosecond high-brightness $\mathrm{KrF}$ laser system. This is supported by the fact that we succeeded in generating $\sim 700$-fs pulses with 8-mJ energy (ASE $<3 \%$ ) with double-pass amplification when the full cross section of our discharge-pumped amplifier was used, and by adding an e-beam-pumped $\mathrm{ArF}$ excimer power amplifier to the system, an output pulse energy of $60 \mathrm{~mJ}$ was achieved. ${ }^{9}$

In conclusion, we have measured the gain characteristics of a discharge-pumped ArF excimer amplifier by using $\sim 700$-fs pulses generated by a dispersively compensated sum-frequency mixing scheme. The small-signal gain and the saturation energy density have been found to be $0.17 \mathrm{~cm}^{-1}$ and $3.65 \mathrm{~mJ} / \mathrm{cm}^{2}$, respectively. The maximum energy density extracted from the saturated amplifier was $10 \mathrm{~mJ} / \mathrm{cm}^{2}$. Since the demonstrated gain characteristics of $\mathrm{ArF}$ compare very well with those of $\mathrm{KrF}$, it seems to be feasible that terawatt-class femtosecond laser systems can now be designed for $193 \mathrm{~nm}$.

The support of the Robert Welch Foundation grant C-0586 and the U.S. Office of Naval Research is gratefully acknowledged. G. Szabó also acknowledges support from the OTKA Foundation of Hungarian Academy of Sciences grant 3056 and National Science Foundation grant INT-9020541.

\section{References}

1. A. J. Taylor, C. R. Tallman, J. P. Roberts, C. S. Lester, T. R. Gosnell, P. H. Y. Lee, and G. A. Kyrala, Opt. Lett. 15, 39 (1990).

2. A. Endoh, M. Watanabe, N. Sarukura, and S. Watanabe, Opt. Lett. 14, 353 (1989).

3. Th. Hofmann, T. E. Sharp, C. B. Dane, P. J. Wisoff, W. L. Wilson, F. K. Tittel, and G. Szabó, IEEE J. Quantum Electron. 28, 1366 (1992).

4. H. Egger, T. S. Luk, K. Boyer, D. F. Muller, H. Pummer, T. Srinivasan, and C. K. Rhodes, Appl. Phys. Lett. 41, 1032 (1982).

5. S. Szatmari and F. P. Schäfer, J. Opt. Soc. Am. B 6, 1877 (1989).

6. J. H. Glownia, M. Kaschke, and P. Sorokin, Opt. Lett. 17, 337 (1992).

7. I. V. Tomov, T. Anderson, and P. M. Rentzepis, Appl. Phys. Lett. 61, 1157 (1992).

8. Th. Hofmann, K. Mossavi, F. K. Tittel, and G. Szabó, Opt. Lett. 17, 1691 (1992).

9. K. Mossavi, Th. Hofmann, F. K. Tittel, and G. Szabó, "Ultrahigh-brightness, femtosecond ArF excimer laser system," Appl. Phys. Lett. (to be published).

10. A. J. Taylor, R. B. Gibson, and J. P. Roberts, Opt. Lett. 13, 814 (1988).

11. P. Simon, H. Gerhardt, and S. Szatmari, Opt. Lett. 14, 1207 (1989).

12. K. Hata, M. Watanabe, and S. Watanabe, Appl. Phys. B 50, 55 (1990).

13. A. J. Taylor, T. R. Gosnell, and J. P. Roberts, Opt. Lett. 15, 118 (1990).

14. S. Szatmari and F. P. Schäfer, J. Opt. Soc. Am. B 4, 1943 (1987).

15. T. H. Dunning and P. J. Hay, J. Chem. Phys. 69, 134 (1978).

16. C. Momma, H. Eichmann, H. Jacobs, A. Tünnermann, H. Welling, and B. Wellegehausen, "Short-pulse amplification and gain dynamics of the ArF excimer laser," Opt. Lett. (to be published). 